\title{
EPHEMERIS EPIGRAPHICA
}

\section{CORPORIS INSCRIPTIONVM LATINARVM SVPPLEMENTVM}

EDITA IVSSV

INSTITVTI ARCHAEOLOGICI ROMANI

VOL. V

ACCEDIT TABVLA GEOGRAPHICA

VENIT

ROMAE APVD INSTITVTVM

BEROLINI APVD GEORGIVM REIMERVM

1884 
